\title{
Use of Super-resolution Immunofluorescence Microscopy to Analyze Tight Junction Protein Interactions in situ
}

\author{
Michael Koval $^{1,2,3}$, Samuel A. Molina ${ }^{1,3}$ and Barbara Schlingmann ${ }^{1}$ \\ ${ }^{1}$ Division of Pulmonary, Allergy, Critical Care and Sleep Medicine, Department of Medicine, Emory \\ University, Atlanta, GA US \\ ${ }^{2}$ Department of Cell Biology, Emory University, Atlanta, GA US \\ ${ }^{3}$ Emory+Children's Center for Cystic Fibrosis and Airways Disease Research, Emory University School \\ of Medicine and Children's Healthcare of Atlanta, Atlanta, GA US
}

Sites of intercellular contact are regulated by structures known as junctions. Among the several classes of intercellular junctions are tight junctions that enable epithelia to form barriers by controlling the permeability of soluble molecules, water and ions across extracellular contacts between cells [1]. Tight junctions are composed of a complex of transmembrane proteins linked to the actin cytoskeleton by cytoplasmic scaffold proteins. Paracellular permeability is directly controlled by transmembrane proteins called claudins [2]. There are about two dozen different human claudin genes; tissue-specific expression of claudins confers distinct permeability to different epithelia. Most claudins directly bind to the scaffold protein zonula occludens-1 (ZO-1) that, in turn, binds to cortical actin which promotes tight junction formation [3]. This is just one of the many protein-protein interactions that are required to form fully functional junctions.

Defining how different tight junction proteins interact to regulate paracellular permeability is an active area of investigation. Most critically, tight junction protein interactions are not easily studied using standard biochemistry [4]. Claudin-claudin interactions are particularly sensitive to detergents [5] and it is difficult to study them in a manner analogous to that used for other classes of junction proteins (e.g. connexins) [6]. Also, solution biochemistry requires disrupting the native functional context of tight junction proteins [7,8]. Thus, we turned to super-resolution immunofluorescence microscopy as a method to understand the molecular basis for tight junction protein interactions in barrier function.

A major research effort by our group is the study of tight junctions in a class of lung cells known as alveolar epithelial cells (AECs) [9]. AECs are large, squamous cells that are critical to prevent the leakage of fluid into the terminal airspaces of the lung, a condition known as acute respiratory distress syndrome (ARDS) [10]. We used STochastic Optical Reconstruction Microscopy (STORM) [11,12] to map the fine structure of tight junctions using primary rat AECs cultured in Transwells to form a functional model alveolar barrier. The cells were fixed and stained for claudin-18 and ZO-1, then imaged. Using STORM, we found that junctional claudin-18 and ZO-1 formed discrete classes of features (plaques), ranging in diameter from $\sim 25 \mathrm{~nm}$ to $\sim 95 \mathrm{~nm}$. Also, the extent of claudin-18/ZO-1 colocalization was not absolute, since there were areas where claudin-18 plaques lacked ZO-1 and vice versa. This contrasts with the more continuous appearance of junction proteins observed by conventional fluorescence microscopy.

These data suggest a previously unappreciated level of heterogeneity in AEC tight junctions. Current efforts are focusing on high resolution imaging of other AEC tight junction proteins under normal conditions in comparison with AECs predisposed for barrier failure. A long term goal is identifying junction proteins that are suitable pharmacologic targets to promote lung barrier function [13]. 
References:

[1] CM Van Itallie, and JM Anderson, Semin Cell Dev Biol 36C (2014), p. 157.

[2] D Gunzel, and AS Yu, Physiol Rev 93 (2013), p. 525.

[3] K Umeda, J Ikenouchi, S Katahira-Tayama, K Furuse, H Sasaki, M Nakayama, T Matsui, S Tsukita, and M Furuse, Cell 126 (2006), p. 741.

[4] F Liu, M Koval, S Ranganathan, S Fanayan, WS Hancock, EK Lundberg, RC Beavis, L Lane, P Duek, L McQuade, NL Kelleher, and MS Baker, J Proteome Res 15 (2016), p. 339.

[5] LL Mitic, VM Unger, and JM Anderson, Protein Sci 12 (2003), p. 218.

[6] S Das, TD Smith, JD Sarma, JD Ritzenthaler, J Maza, BE Kaplan, LA Cunningham, L Suaud, MJ Hubbard, RC Rubenstein, and M Koval, Mol Biol Cell 20 (2009), p. 2593.

[7] M Koval, Tissue Barriers 1 (2013), p. e24518.

[8] A Lingaraju, TM Long, Y Wang, JR Austin, 2nd, and JR Turner, Semin Cell Dev Biol 42 (2015), p. 13.

[9] B Schlingmann, SA Molina, and M Koval, Semin Cell Dev Biol 42 (2015), p. 47.

[10] LB Ware, and MA Matthay, Am J Respir Crit Care Med 163 (2001), p. 1376.

[11] MJ Rust, M Bates, and X Zhuang, Nat Methods 3 (2006), p. 793.

[12] DT Burnette, P Sengupta, Y Dai, J Lippincott-Schwartz, and B Kachar, Proc Natl Acad Sci U S A 108 (2011), p. 21081.

[13] Supported by NIH R01-HL116958 (MK), NIH T32-AA-013528 (SAM), the German Academic Exchange Service (DAAD) (BS), Emory+Children's Center of Excellence for Cystic Fibrosis Research (MK) and the Emory University Integrated Cellular Imaging Microscopy Core of the Winship Cancer Institute Comprehensive Cancer Center Grant NIH P30CA138292.
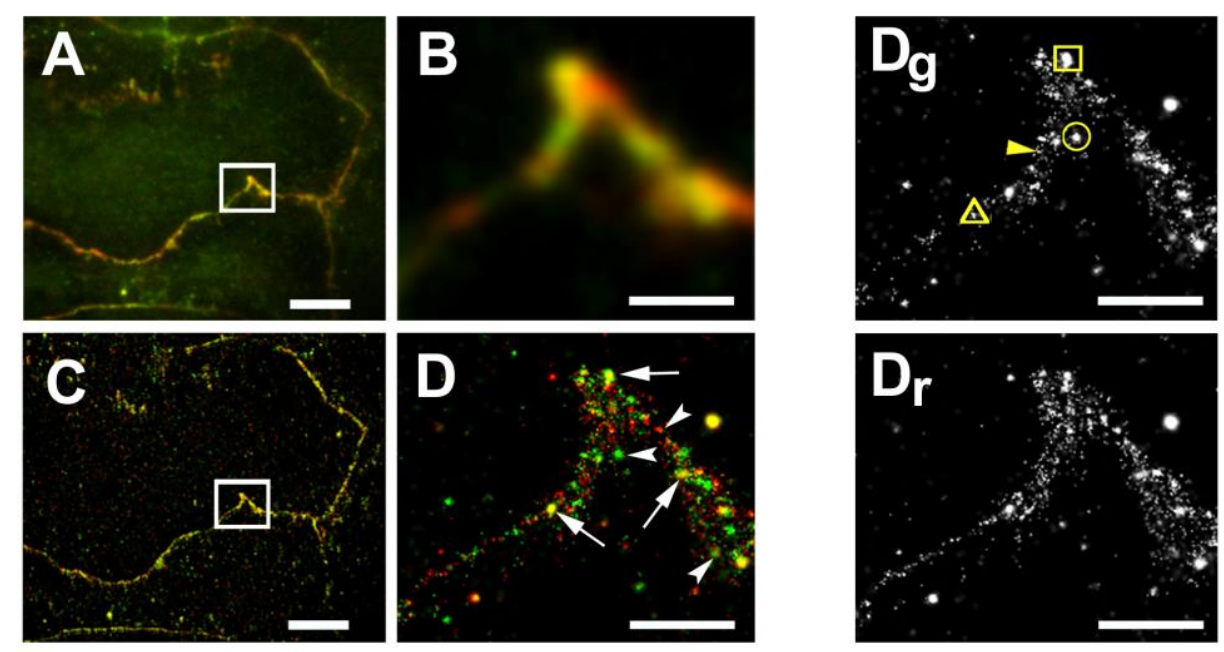

Figure 1. Primary rat AECs were double-immunolabeled for claudin-18 (green) and ZO-1 (red), then imaged by standard wide field confocal microscopy (A,B) or STORM (C,D). Panels A,C show a large field image (Bar, $10 \mathrm{um}$ ), the remaining panels show a magnified portion as indicated by the square in A,C (Bar, 1 micron). D. Plaques containing both claudin-18 and ZO-1 are denoted by arrows, single labeled plaques are indicated by arrowheads. Dg and Dr are the green (claudin-18) and red (ZO-1) channels of panel D, respectively. Feature classes with different diameter are labeled by the yellow square $(90-100 \mathrm{~nm})$, circle $(65-85 \mathrm{~nm})$, triangle $(35-45 \mathrm{~nm})$ and arrowhead $(25 \mathrm{~nm})$. 